\title{
The Effect of Obesity on Response to Neoadjuvant Therapy in Locally Advanced Gastric Cancer
}

\author{
Aysegul Sakin ${ }^{1 *}$, Suleyman Sahin², Abdullah Sakin³, Mehmet Naci Aldemir³, \\ Irfan Bayram ${ }^{4}$, Cetin Kotan ${ }^{5}$
}

\begin{abstract}
Introduction: The effect of obesity on response to neoadjuvant chemotherapy (NACT) remains unknown. We aimed to investigate the effect of obesity on response to NACT and survival in locally-advanced gastric cancer (GC). Methods: From 2010 to 2019, 142 GC patients with clinical stage III disease who underwent curative surgery after NACT were enrolled. Patients were divided into 3 groups according to body mass index (BMI) as follows; BMI $<25$ $\mathrm{kg} / \mathrm{m}^{2}, \mathrm{BMI}=25-30 \mathrm{~kg} / \mathrm{m}^{2}$, and BMI $>30 \mathrm{~kg} / \mathrm{m}^{2}$. The Mandard tumor regression grading system was used for tumor regression grade (TRG). Results: Of the $142 \mathrm{GC}$ patients, $45(31.7 \%)$ were female. The median age was 58 years. BMI was $<25 \mathrm{~kg} / \mathrm{m}^{2}$ in $60(42.3 \%)$ patients, $25-30 \mathrm{~kg} / \mathrm{m} 2$ in $44(31 \%)$ patients, and $>30 \mathrm{~kg} / \mathrm{m}^{2}$ in $38(26.8 \%)$ patients. The numbers of patients with TRGI-II, TRGIII, and TRGIV-V were 35 (24.6\%), 44 (31\%), and 63 (44.4\%), respectively. There was no statistically significant difference among BMI groups in terms of disease-free survival (DFS) and overall survival (OS) ( $\mathrm{p}=0.919$ and $\mathrm{p}=0.398$, respectively). According to TRG groups; mDFS was 46 months in TRG I-II, 28 months in TRG III, and 18 months in TRG IV-V ( $<<0.001)$. In multivariate analysis, presence of perineural invasion and lymphovascular invasion were the factors affecting TRG. Conclusion: In our study, we found that pre-treatment obesity did not affect the TRG in clinical stage III GC patients. However, a better TRG status was associated with improved survival.
\end{abstract}

Keywords: Obesity- gastric cancer- tumor regression grade- body mass index

Asian Pac J Cancer Prev, 21 (9), 2723-2731

\section{Introduction}

Gastric cancer (GC) remains a major cause of cancer-related deaths globally, with high mortality rates even in the early stages. In population-based series, the 5 -year survival rate for completely-resected stage I-II GC patients is approximately $35-75 \%$ (Ferlay et al., 2015; Siegel et al., 2019). Multidisciplinary team approach is the standard of care in the treatment of GC. Randomized trials and meta-analyses have indicated a significant survival benefit with adjuvant chemoradiotherapy (CRT), Neoadjuvant chemotherapy (NACT), and adjuvant chemotherapy (ACT) for locally-advanced GC patients, as compared with surgery alone (Cunningham et al., 2006; Xiong et al., 2014; Al-Batran et al., 2019).

NACT prior to surgery can prompt tumor shrinkage, decrease intraoperative spread, and increase the rate of R0 resection during surgery. Neoadjuvant treatment evaluates the effect of NACT regimen as well as guiding the postoperative treatment approach. Moreover, if metastatic spread occurs during or after NACT, particularly in patients who have a greater risk of developing distant metastases, an unnecessary surgery will be prevented.The responses to frequently-used chemotherapy (CT) regimens range from $49 \%$ to $69.7 \%$ (Kobayashi and Kimura, 2000; Cunningham et al., 2006).

The rate of (pathological complete response) pCR in GC after NACT is relatively low. Previous studies and meta-analysis have reported that pCR increases survival (Lorenzen et al., 2013; Li et al., 2018). However, it is difficult to define which patient effectively responds to NACT. The ability to predict the pathological tumor response before treatment can provide a significant clinical advantage, provide additional information to allow tailored ACT options, and help evaluate the individual prognosis (Melcher et al., 1996; Li et al., 2012; Al-Batran et al., 2016).

Obesity is an increasing global health problem. Body mass index (BMI), calculated by the patient's weight and height, is a good way to measure obesity. Moreover, BMI

${ }^{1}$ Department of Internal medicine, University of Health Sciences, Van Research and Training Hospital, Van, Turkey. ${ }^{2}$ Department of Medical Oncology, University of Health Sciences, Van Research and Training Hospital, Van, Turkey. ${ }^{3}$ Department of Medical Oncology, Yuzuncu Yil University Medical School, 65030, Van, Turkey. ${ }^{4}$ Department of Pathology, Yuzuncu Yil University Medical School, 65030, Van, Turkey. ${ }^{5}$ Department of General surgery, Yuzuncu Yil University Medical School, 65030, Van, Turkey. *For Correspondence: mdaysegulsakin@gmail.com 
is an effective method in evaluating the nutritional status of cancer patients (Liedman et al., 1996; Mokdad et al., 2003). Many studies have shown that obesity is associated with poor surgical outcomes in cancer patients, including GC (Bege et al., 2009; Benns et al., 2009; Kunisaki et al., 2009).

In previous studies, the relation of obesity with postoperative complications and survival was examined (Dhar et al., 2000; Tsujinaka et al., 2007; Kunisaki et al., 2009; Kunisaki, 2010). However, the effect of obesity on response to NACT remains unknown. In the present study, we aim to investigate the effect of obesity on response to treatment and long-term survival in clinical stage III GC patients treated with NACT.

\section{Materials and Methods}

\section{Study population}

From 2010 through 2019, all patients with locallyadvanced GC, who underwent NACT followed by gastrectomy in Van Yüzüncü y1l University Hospital, were analyzed retrospectively. The inclusion criteria were defined as follows; age $\geq 18$ years and having received NACT for locally-advanced (clinical stage III) GC. Patients with any of the following criteria were excluded from the study; age $<18$ years, those not undergoing surgery, metastatic disease, history of a second primary cancer, histologic subtypes other than adenocarcinoma (AC), [e.g., Signet ring cell carcinoma (SRCC), and Mucinous adenocarcinoma (MAC)], patients who died due to surgical complications, and those with missing data. The clinical stage of the patients was determined by computed tomography taken before treatment. Patients were restaged according to the AJCC (American Joint Committee on Cancer) Cancer Staging Manual, 8th edition.

\section{Data collection}

Demographic data of the patients including gender, age, Eastern Cooperative Oncology Group performance scale (ECOG PS), height, weight, presence of hypertension (HT) or diabetes mellitus (DM), smoking status, clinical stage, Lauren classification, primary tumor localization, histology (AC, MAC, and SRCC), tumor grade, neoadjuvant regimen, type of surgery (subtotal, total gastrectomy, D1, or D2 dissection), ypTNM stage, pathological tumor stage (ypT), pathological lymph node stage (ypN), presence of lymphovascular (LVI) and perineural invasion (PNI), tumor regression grade(TRG), human epidermal growth factor receptor 2 (HER-2) status, adjuvant regimen, recurrence status, site of recurrence, and final status were obtained from the written archive files. Patients were divided into 3 groups according to BMI as follows; $\mathrm{BMI}<25 \mathrm{~kg} / \mathrm{m}^{2}, \mathrm{BMI}=25-30 \mathrm{~kg} / \mathrm{m}^{2}$, and BMI $>30 \mathrm{~kg} / \mathrm{m}^{2}$. In this study, no classification such as BMI $<18.5 \mathrm{~kg} / \mathrm{m}^{2}$ as a separate group could be made since there were only 3 patients with BMI $<18.5 \mathrm{~kg} / \mathrm{m}^{2}$.

\section{Response to treatment}

The Mandard tumor regression grading system was used for TRG, which was defined as follows; TRG I= Complete regression, fibrosis with no evidence of tumor cells in the specimen, TRG II= Fibrosis and rare residual tumor cells in the specimen, TRG II= Fibrosis outgrowing residual tumor in the specimen, TRG IV=Rare fibrosis and residual tumor outgrowing fibrosis, and $\mathrm{TRG} V=$ Tumor without evidence of regressive changes. The patients were divided into 2 groups according to TRG status; Group 1= TRG I-II and group 2= TRG III-IV-V.

\section{Follow-up}

Disease-free survival (DFS) was calculated from the date of diagnosis to the date of progression or last followup. Overall survival (OS) was calculated from the date of diagnosis to the date of death or last follow-up.

\section{Ethics committee approval}

This study was conducted in accordance with the Declaration of Helsinki and it was reviewed and approved by the Ethics Committee of the Van Yüzüncü Y1l University Faculty of Medicine (2020/03-52).

\section{Statistical analysis}

Statistical Package for Social Sciences 22.0 for Windows software (Armonk NY, IBM Corp. 2013) was used for all statistical analysis. Student's t test was used when the numerical variable provided the normal distribution condition in two independent groups, whereas Mann Whitney U test was used when the normal distribution condition was not provided. Chi-square analysis was used to compare the ratios in the groups. Survival analyzes were performed by Kaplan Meier Analysis. For the determinant factors, logistic regression analysis was used. Statistical significance level was accepted as $\mathrm{p}<0.05$.

\section{Results}

\section{Clinicopathological characteristics}

Of the 142 GC patients, $45(31.7 \%)$ were female and $97(68.3 \%)$ were male. The median age was 58 years (range, $31-79$ ). BMI was $<25 \mathrm{~kg} / \mathrm{m}^{2}$ in $60(42.3 \%$ ) patients, $25-30 \mathrm{~kg} / \mathrm{m}^{2}$ in $44(31 \%)$ patients, and $>30 \mathrm{~kg} / \mathrm{m}^{2}$ in 38 $(26.8 \%)$ patients. Twelve $(8.5 \%)$ patients had DM and 26 (18.3\%) patients had HT. Sixty-nine (48.6\%) patients were smokers. According to the Lauren classification, tumor was intestinal type in $120(84.5 \%)$ patients. In $101(71.1 \%)$ patients, histological subtype was AC. The tumor grade in $41(29.3 \%)$ patients was 3 . As a surgical procedure, $119(83.8 \%)$ patients underwent total gastrectomy and 94 (66.2\%) patients had D1 dissection. PNI was present in $77(54.2 \%)$ patients and LVI was positive in $66(46.5 \%)$ patients. During the median follow-up time of 15 months, $52(36.6 \%)$ patients developed recurrence and 23 (16.2\%) patients died (Table 1).

\section{Treatment regimens}

Considering the NACT regimens, $52(36.6 \%)$ patients received either epirubicin + oxaliplatine + capecitabine (EOX), epirubicin + oxaliplatine + fluorouracil (EOF), epirubicin + cisplatin + capecitabine (ECX), or epirubicin + cisplatin + fluorouracil (ECF), 27 (19\%) patients received either docetaxel + cisplatin + fluorouracil (DCF) 
Table 1. Patient Characteristics and Clinicopathological Features in BMI Groups

\begin{tabular}{|c|c|c|c|c|c|c|c|c|c|c|}
\hline \multirow[t]{2}{*}{ Variable } & & \multicolumn{2}{|c|}{$\begin{array}{l}\text { Total Patients } \\
\quad(\mathrm{n}=142)\end{array}$} & \multicolumn{2}{|c|}{$\begin{array}{c}\mathrm{BMI}<25 \mathrm{~kg} / \mathrm{m}^{2} \\
(\mathrm{n}=60)\end{array}$} & \multicolumn{2}{|c|}{$\begin{array}{c}\mathrm{BMI}=25-30 \mathrm{~kg} / \\
\mathrm{m}^{2}(\mathrm{n}=42)\end{array}$} & \multicolumn{2}{|c|}{$\begin{array}{c}\mathrm{BMI}>30 \mathrm{~kg} / \mathrm{m}^{2} \\
\quad(\mathrm{n}=38)\end{array}$} & \multirow[t]{2}{*}{$\mathrm{p}$} \\
\hline & & $\mathrm{n}$ & $\%$ & $\mathrm{n}$ & $\%$ & $\mathrm{n}$ & $\%$ & $\mathrm{n}$ & $\%$ & \\
\hline \multirow[t]{2}{*}{ Gender } & Female & 45 & 31.7 & 13 & 21.7 & 12 & 27.3 & 20 & 52.6 & 0.004 \\
\hline & Male & 97 & 68.3 & 47 & 78.3 & 32 & 72.7 & 18 & 47.4 & \\
\hline \multirow[t]{2}{*}{ Age (year) } & Median & 58 & & 60 & & 57 & & 59 & & 0.192 \\
\hline & $(\min -\max )$ & $(31-79)$ & & $(21-79)$ & & $(44-77)$ & & $(42-71)$ & & \\
\hline \multirow[t]{2}{*}{ ECOG PS } & 0 & 125 & 88 & 51 & 85 & 39 & 88.6 & 35 & 92.1 & 0.566 \\
\hline & 1 & 17 & 12 & 9 & 15 & 5 & 11.4 & 3 & 7.9 & \\
\hline Height & $\mathrm{cm}$ & $165.2 \pm 9.1$ & & $167.5 \pm 8.2$ & & $166.0 \pm 9.0$ & & $159.6 \pm 8.6$ & & 0.001 \\
\hline Weight & $\mathrm{kg}$ & $71.5 \pm 12.5$ & & $61.1 \pm 9.1$ & & $73.7 \pm 7.9$ & & $82.5 \pm 9.8$ & & $<0.001$ \\
\hline BMI & $\mathrm{Kg} / \mathrm{m}^{2}$ & $26.29 \pm 4.71$ & & $21.6 \pm 2.3$ & & $26.9 \pm 1.0$ & & $32.2 \pm 2.1$ & & $<0.001$ \\
\hline Hypertension & Yes & 26 & 18.3 & 14 & 23.3 & 7 & 15.9 & 5 & 13.2 & 0.395 \\
\hline Diabetes mellitus & Yes & 12 & 8.5 & 3 & 5 & 4 & 9.1 & 5 & 13.2 & 0.381 \\
\hline Smoking status & Yes & 69 & 48.6 & 34 & 56.7 & 21 & 47.7 & 14 & 36.8 & 0.159 \\
\hline \multirow[t]{2}{*}{ Lauren classification } & Intestinal & 120 & 84.5 & 46 & 76.7 & 38 & 86.4 & 36 & 94.7 & 0.051 \\
\hline & Diffuse & 22 & 15.5 & 14 & 23.3 & 6 & 13.6 & 2 & 5.3 & \\
\hline \multirow[t]{5}{*}{ Location } & GEJ & 8 & 5.6 & 6 & 10 & 0 & 0 & 2 & 5.3 & 0.101 \\
\hline & Cardia & 61 & 43.0 & 27 & 45 & 17 & 38.6 & 17 & 44.7 & \\
\hline & Body & 32 & 22.5 & 15 & 25 & 11 & 25 & 6 & 15.8 & \\
\hline & Antrum & 37 & 26.1 & 9 & 15 & 16 & 36.4 & 12 & 31.6 & \\
\hline & Linitis plastica & 4 & 2.8 & 3 & 5 & 0 & 0 & 1 & 2.6 & \\
\hline \multirow[t]{3}{*}{ Histology } & $\mathrm{SRCC}$ & 24 & 16.9 & 11 & 18.3 & 9 & 20.5 & 4 & 10.5 & 0.738 \\
\hline & $\mathrm{AC}$ & 101 & 71.1 & 42 & 70 & 29 & 65.9 & 30 & 78.9 & \\
\hline & MAC & 17 & 12.0 & 7 & 11.7 & 6 & 13.6 & 4 & 10.5 & \\
\hline \multirow[t]{3}{*}{ Grade } & I & 12 & 8.6 & 3 & 5.2 & 4 & 9.1 & 5 & 13.2 & 0.68 \\
\hline & II & 87 & 62.1 & 39 & 67.2 & 26 & 59.1 & 22 & 57.9 & \\
\hline & III & 41 & 29.3 & 16 & 27.6 & 14 & 31.8 & 11 & 28.9 & \\
\hline \multirow[t]{3}{*}{ NACT regimen } & ECF-ECX- EOF-EOX & 52 & 36.6 & 14 & 23.3 & 15 & 34.1 & 23 & 60.5 & 0.01 \\
\hline & DCF-DCX & 27 & 19.0 & 10 & 16.7 & 13 & 29.5 & 4 & 10.5 & \\
\hline & FLOT & 63 & 44.4 & 36 & 60 & 16 & 36.4 & 11 & 28.9 & \\
\hline NACT cycle no. & & $3.8 \pm 1.4$ & & $3.7 \pm 1.1$ & & $3.9 \pm 1.1$ & & $3.7 \pm 2.3$ & & 0.082 \\
\hline \multirow[t]{2}{*}{ Gastrectomy } & Subtotal & 23 & 16.2 & 7 & 11.7 & 9 & 20.5 & 7 & 18.4 & 0.422 \\
\hline & Total & 119 & 83.8 & 53 & 88.3 & 35 & 79.5 & 31 & 81.6 & \\
\hline \multirow[t]{2}{*}{ Lymphadenectomy } & D1 & 94 & 66.2 & 40 & 66.7 & 32 & 72.7 & 22 & 57.9 & 0.365 \\
\hline & D2 & 48 & 33.8 & 20 & 33.3 & 12 & 27.3 & 16 & 42.1 & \\
\hline \multirow[t]{2}{*}{ Surgical margin } & Positive & 132 & 93.0 & 53 & 88.3 & 42 & 95.5 & 37 & 97.4 & 0.218 \\
\hline & Positive & 10 & 7.0 & 7 & 11.7 & 2 & 4.5 & 1 & 2.6 & \\
\hline ypTNM & 0 & 12 & 8.5 & 4 & 6.7 & 5 & 11.4 & 3 & 7.9 & 0.776 \\
\hline & 1 & 18 & 12.7 & 7 & 11.7 & 6 & 13.6 & 5 & 13.2 & \\
\hline & 2 & 36 & 25.4 & 14 & 23.3 & 9 & 20.5 & 13 & 34.2 & \\
\hline & 3 & 76 & 53.5 & 35 & 58.3 & 24 & 54.5 & 17 & 44.7 & \\
\hline ypT & 0 & 12 & 8.5 & 4 & 6.7 & 5 & 11.4 & 3 & 7.9 & 0.722 \\
\hline & 1 & 17 & 12.0 & 5 & 8.3 & 5 & 11.4 & 7 & 18.4 & \\
\hline & 2 & 11 & 7.7 & 5 & 8.3 & 3 & 6.8 & 3 & 7.9 & \\
\hline & 3 & 70 & 49.3 & 31 & 51.7 & 19 & 43.2 & 20 & 52.6 & \\
\hline & 4 & 32 & 22.5 & 15 & 25 & 12 & 27.3 & 5 & 13.2 & \\
\hline ypN & 0 & 45 & 31.7 & 19 & 31.7 & 15 & 34.1 & 11 & 28.9 & 0.423 \\
\hline & 1 & 30 & 21.1 & 10 & 16.7 & 10 & 22.7 & 10 & 26.3 & \\
\hline & 2 & 22 & 15.5 & 9 & 15 & 4 & 9.1 & 9 & 23.7 & \\
\hline & 3 & 45 & 31.7 & 22 & 36.7 & 15 & 34.1 & 8 & 21.1 & \\
\hline No. of nodes removed & & $27.3 \pm 13.1$ & 9-78) & $24.6 \pm 11.4$ & & $26.6 \pm 11.2$ & & $31.4 \pm 15.0$ & & 0.13 \\
\hline No. of nodes positive & & $5.05 \pm 6.90$ & $0-35)$ & $5.1 \pm 6.1$ & & $5.2 \pm 7.9$ & & $4.1 \pm 5.4$ & & 0.703 \\
\hline PNI & Presence & 77 & 54.2 & 31 & 51.7 & 25 & 56.8 & 21 & 55.3 & 0.869 \\
\hline
\end{tabular}


Table 1. Continued

\begin{tabular}{|c|c|c|c|c|c|c|c|c|c|c|}
\hline \multirow[t]{2}{*}{ Variable } & & \multicolumn{2}{|c|}{$\begin{array}{l}\text { Total Patients } \\
\quad(\mathrm{n}=142)\end{array}$} & \multicolumn{2}{|c|}{$\begin{array}{c}\mathrm{BMI}<25 \mathrm{~kg} / \mathrm{m}^{2} \\
(\mathrm{n}=60)\end{array}$} & \multicolumn{2}{|c|}{$\begin{array}{c}\mathrm{BMI}=25-30 \mathrm{~kg} / \\
\mathrm{m}^{2}(\mathrm{n}=42)\end{array}$} & \multicolumn{2}{|c|}{$\begin{array}{c}\text { BMI }>30 \mathrm{~kg} / \mathrm{m}^{2} \\
(\mathrm{n}=38)\end{array}$} & \multirow[t]{2}{*}{$\mathrm{p}$} \\
\hline & & $\mathrm{n}$ & $\%$ & $\mathrm{n}$ & $\%$ & $\mathrm{n}$ & $\%$ & $\mathrm{n}$ & $\%$ & \\
\hline LVI & Presence & 66 & 46.5 & 29 & 48.3 & 20 & 45.5 & 17 & 44.7 & 0.929 \\
\hline \multirow[t]{4}{*}{ HER-2 status } & 0 & 108 & 76.1 & 43 & 71.7 & 36 & 81.8 & 29 & 76.3 & 0.752 \\
\hline & 1 & 12 & 8.5 & 6 & 10 & 3 & 6.8 & 3 & 7.9 & \\
\hline & 2 & 12 & 8.5 & 6 & 10 & 4 & 9.1 & 2 & 5.3 & \\
\hline & 3 & 10 & 7 & 5 & 8.3 & 1 & 2.3 & 4 & 10.5 & \\
\hline \multirow[t]{5}{*}{ TRG } & I & 12 & 8.5 & 4 & 6.7 & 5 & 11.4 & 3 & 7.9 & 0.962 \\
\hline & II & 23 & 16.2 & 10 & 16.7 & 6 & 13.6 & 7 & 18.4 & \\
\hline & III & 44 & 31 & 17 & 28.3 & 13 & 29.5 & 14 & 36.8 & \\
\hline & IV & 58 & 40.8 & 27 & 45 & 18 & 40.9 & 13 & 34.2 & \\
\hline & $\mathrm{V}$ & 5 & 3.5 & 2 & 3.3 & 2 & 4.5 & 1 & 2.6 & \\
\hline \multirow[t]{2}{*}{ TRG groups } & Group 1 & 35 & 26.3 & 14 & 23.3 & 11 & 25 & 10 & 26.3 & 0.944 \\
\hline & Group 2 & 107 & 73.7 & 46 & 76.7 & 33 & 75 & 28 & 73.6 & \\
\hline \multirow[t]{4}{*}{ ACT regimen } & XELOX-FOLFOX-CF & 35 & 24.6 & 19 & 31.7 & 10 & 22.7 & 6 & 15.8 & 0.045 \\
\hline & EOX-EOF-ECX-ECF & 43 & 30.3 & 12 & 20 & 12 & 27.3 & 19 & 50 & \\
\hline & DCF-DCX & 21 & 14.8 & 7 & 11.7 & 9 & 20.5 & 5 & 13.2 & \\
\hline & FLOT & 43 & 30.3 & 22 & 36.7 & 13 & 29.5 & 8 & 21.1 & \\
\hline \multirow{8}{*}{$\begin{array}{l}\text { Recurrence and localiza- } \\
\text { tion }\end{array}$} & Yes & 52 & 36.6 & 21 & 35 & 19 & 43.2 & 12 & 31.6 & 0.552 \\
\hline & Locoregional & 2 & 3.8 & 0 & 0 & 1 & 5.3 & 1 & 8.3 & 0.281 \\
\hline & Liver & 16 & 30.8 & 5 & 23.8 & 7 & 36.8 & 4 & 33.3 & \\
\hline & Peritoneum & 20 & 38.5 & 7 & 33.3 & 8 & 42.1 & 5 & 41.7 & \\
\hline & Distant Ln & 6 & 11.5 & 2 & 9.5 & 2 & 10.5 & 2 & 16.7 & \\
\hline & Lung & 5 & 9.6 & 5 & 23.8 & 0 & 0 & 0 & 0 & \\
\hline & Brain & 1 & 1.9 & 0 & 0 & 1 & 5.3 & 0 & 0 & \\
\hline & Bone & 2 & 3.8 & 2 & 9.5 & 0 & 0 & 0 & 0 & \\
\hline Last Status & Exitus & 23 & 16.2 & 7 & 11.7 & 11 & 25 & 5 & 13.2 & 0.159 \\
\hline
\end{tabular}

Abbreviations: AC, Adenocarcinoma; ACT, Adjuvant chemotherapy; BMI, Body mass index; CF, Fluorouracil + cisplatin; ECOG PS, Eastern cooperative oncology group performance scale; DCF, Docetaxel + cisplatin + fluorouracil; DCX, Docetaxel + cisplatin + capecitabine; ECF, Epirubicin + cisplatin + fluorouracil; ECX, Epirubicin + cisplatin + capecitabine; EOF, Epirubicin + oxaliplatin + fluorouracil; EOX, Epirubicin + oxaliplatin + capecitabine; FLOT, Fluorouracil + folinic acid + oxaliplatin + docetaxel; FOLFOX, Fluorouracil + folinic acid + oxaliplatin; GEJ, Gastroesophageal junction; HER-2, Human epidermal growth factor receptor 2; PNI, Perineural invasion; SRCC, Signet ring cell carcinoma; LN, Lymph node; LVI, Lymphovascular invasion; MAC, Mucinous adenocarcinoma; NACT, Neoadjuvant chemotherapy; TRG, Tumor regression grade; XELOX, Capecitabine + oxaliplatin; ypT, Pathological tumor stage; ypN, Pathological lymph node stage.

or docetaxel + cisplatin + capecitabine (DCX), and 63 (44.4\%) patients received fluorouracil + folinic acid + oxaliplatine + docetaxel (FLOT). Patients received an average of $3.5 \pm 1.4 \mathrm{CT}$ cycles. All patients were able to receive ACT. As adjuvant regimens; 35 (24.6\%) patients were given either capecitabine + oxaliplatine (XELOX), fluorouracil + folinic acid + oxaliplatine (FOLFOX), or fluorouracil + cisplatin (CF), 43 (30.3\%) patients were
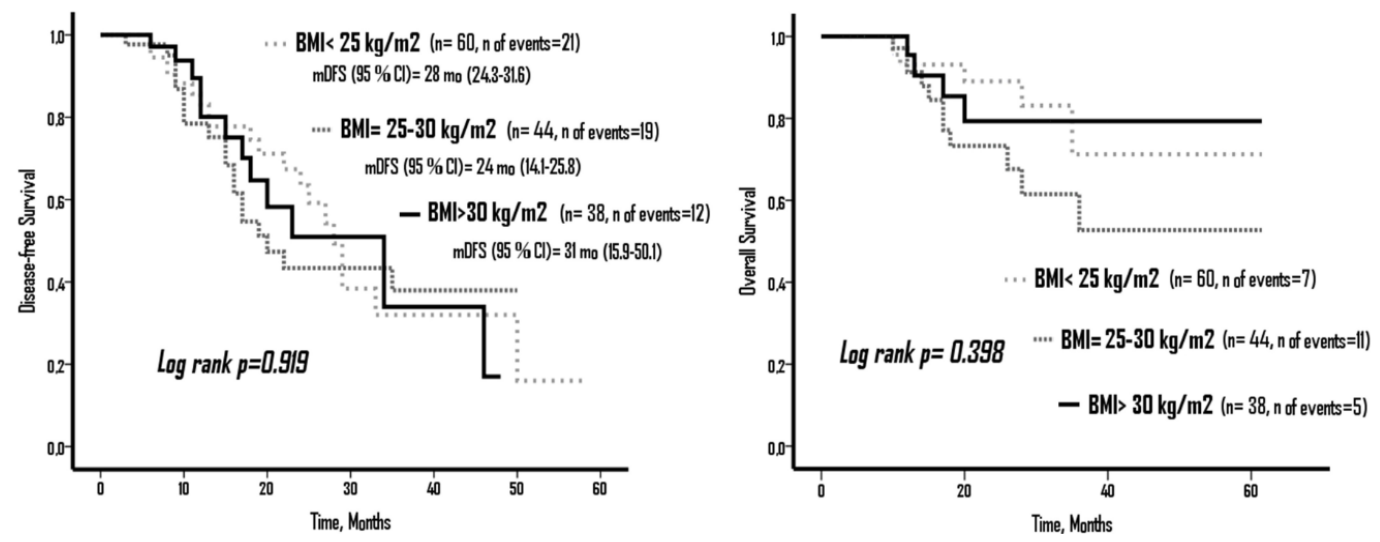

Figure 1. Disease-Free Survival (DFS) and Overall Survival (OS) According to Body Mass Index (BMI) Groups. 
given either EOX, EOF, ECX, or ECF, 21 (14.8\%) patients were given either DCF or DCX, and 43 patients were given FLOT (Table 1).

\section{Clinicopathological features in TRG groups}

Thirty-five (24.6\%) patients were TRG I-II, 44 (31\%) patients were TRG III, and $63(44.4 \%)$ patients were TRG IV-V. There was no significant difference between the TRG groups in terms of age, gender, comorbidity, Lauren classification, tumor localization, tumor grade, NACT regimen, the numbers of NACT cycles, surgical margin, the numbers of lymph nodes removed, HER-2 status, and ACT regimen. There was a statistically significant difference among the groups in terms of ypTNM, ypT, $\mathrm{ypN}$, the number of lymph nodes removed, LVI, PNI, development of recurrence, and exitus rates (Table 2).

\section{Survival analysis}

According to BMI groups, there was no statistically significant difference in terms of DFS and OS $(p=0.919$ and $\mathrm{p}=0.398$, respectively). $\mathrm{mDFS}$ durations in $\mathrm{BMI}<25$ $\mathrm{mg} / \mathrm{kg}^{2}, \mathrm{BMI}=25-30 \mathrm{mg} / \mathrm{kg}^{2}$, and BMI $>30 \mathrm{mg} / \mathrm{kg}^{2}$ were 28 months (95\% confident interval [CI], 24.3-31.6), 24 months (95\% CI, 14.1-25.8), and 31 months (95\% CI, 15.9-50.1), respectively. However, mOS could not be reached (Figure 1)

Based on the TRG groups, there was a statistically significant difference in terms of DFS and OS $(p<0.001$ and $\mathrm{p}=0.001$, respectively). According to TRG groups; mDFS was 46 months in TRG I-II, 28 months $(95 \%$ CI, 9.8-24.1) in TRG III, and 18 months (95\% CI, 12.8-23.1) in TRGIV-V. However, mOS could not be reached (Figure 2).

\section{Factors affecting TRG}

In multivariate analysis with enter model; height, weight, BMI, Lauren classification, histology, and the number of NACT cycles did not affect TRG. However, presence of PNI (Odds ratio[OR],5.3, $95 \%$ CI, 1.1-23.6) and LVI (OR, 25.0, $95 \%$ CI, 4.3-144.4) affected TRG $(p=0.028$ and $p<0.001$, respectively) (Table 3 ).

\section{Discussion}

In this study, we investigated the effect of obesity on response to NACT and long-term survival in clinical stage III GC patients. In addition, we evaluated the prognostic effect of TRG in GC patients with real life data and found that BMI did not affect both TRG and long-term survival. However, we observed that survival was significantly better in those with an increased response to NACT. In addition, the presence of PNI and LVI were determined as the predictive factors affecting TRG.

Previous studies have reported that the obesity affects the response to NACT in various solid tumors such as prostate cancer, rectal cancer, breast cancer, and pancreas cancer (Farr et al., 2017; Park et al., 2017; Duconseil et al., 2019; Sun et al., 2020). Park et al., (2017) reported that obesity reduced the complete response rate by $40 \%$ in their study with rectal cancer patients receiving neoadjuvant CRT. Likewise, another study of pancreatic cancer by Duconseil et al., (2019) has reported that obesity is determined as the factor affecting survival. Similarly, Karatas et al., (2017) has reported that obesity is an independent prognostic factor for $\mathrm{pCR}$, with a poor survival in breast cancer patients who received NACT.

Previous studies regarding GC patients have only investigated the effects of obesity on either post-surgical complications or mortality (Dhar et al., 2000; Kunisaki et al., 2009; Bickenbach et al., 2013; Wong et al., 2014; Palmela et al., 2017). Some of these studies also examined the relationship between obesity and long-term survival (Bickenbach et al., 2013; Wong et al., 2014). Wong et al. reported that obesity may cause technical difficulties to achieving $\mathrm{R} 0$ resection during gastric cancer surgery. However, an increased BMI did not affect DFS or OS (Wong et al., 2014). A study from Memorial Sloan-Kettering Cancer Center conducted by Bickenbach et al. evaluated the impact of obesity on survival in GC patients and reported fewer lymph node dissection rates and higher complication rates in patients with BMI $>25$ $\mathrm{kg} / \mathrm{m}^{2}$; however, survival was similar between the BMI groups (Bickenbach et al., 2013). In our study, patients who died due to surgical complications were not included. Furthermore, in our study, the ratio of number of positive

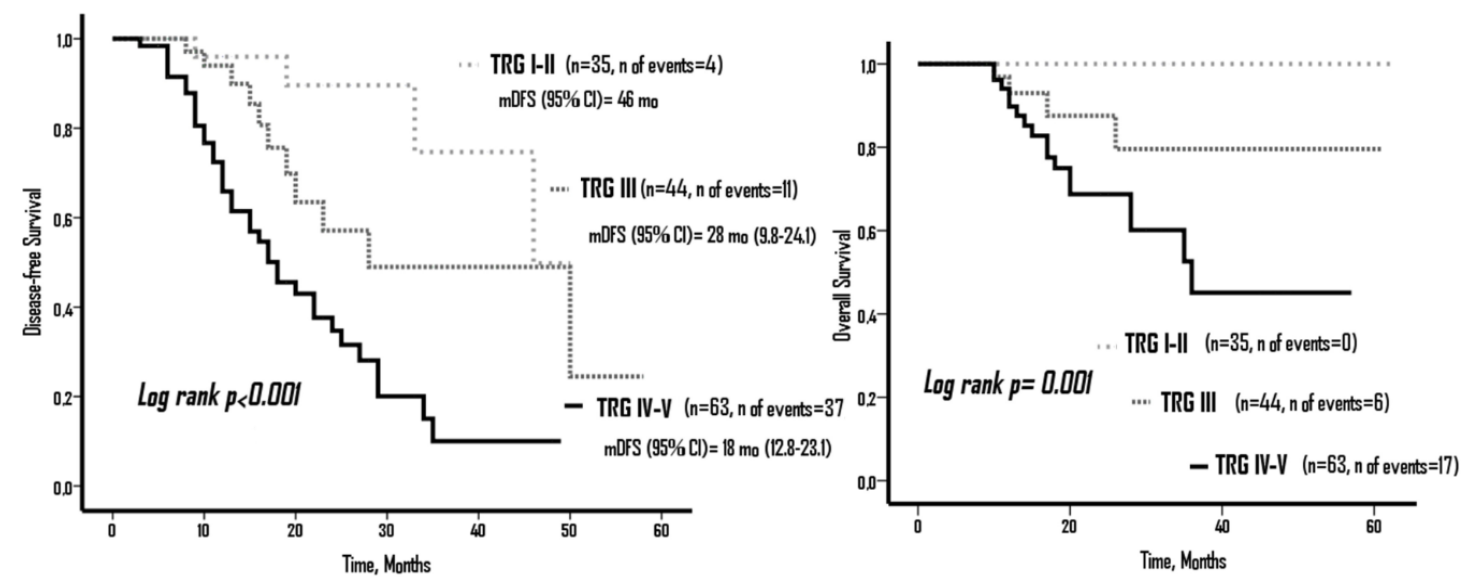

Figure 2. Disease-Free Survival (DFS) and Overall Survival (OS) According to Tumor Regression Grade (TRG) Groups. 
Aysegul Sakin et al

Table 2. Univariate Analysis for TRG Groups (TRG I-II vs. III-IV-V).

\begin{tabular}{|c|c|c|c|c|c|c|}
\hline \multirow[t]{2}{*}{ Variable } & & \multicolumn{2}{|c|}{ Group $1(n=35)$} & \multicolumn{2}{|c|}{ Group $2(\mathrm{n}=107)$} & \multirow[t]{2}{*}{$\mathrm{p}$} \\
\hline & & $\mathrm{n}$ & $\%$ & $\mathrm{n}$ & $\%$ & \\
\hline \multirow[t]{2}{*}{ Gender } & Female & 7 & 20 & 38 & 35.5 & 0.87 \\
\hline & Male & 28 & 80 & 69 & 64.5 & \\
\hline \multirow[t]{2}{*}{ Age (year) } & Median & 61 & & 58 & & 0.192 \\
\hline & $(\min -\max )$ & $(35-79)$ & & $(31-75)$ & & \\
\hline \multirow[t]{2}{*}{ ECOG PS } & 0 & 33 & 94.3 & 92 & 86 & 0.242 \\
\hline & 1 & 2 & 5.7 & 15 & 14 & \\
\hline Height & $\mathrm{cm}$ & $168.09 \pm 9.42$ & & $164.21 \pm 8.89$ & & 0.033 \\
\hline Weight & $\mathrm{kg}$ & $73.74 \pm 13.90$ & & $70.77 \pm 12.08$ & & 0.24 \\
\hline BMI & $\mathrm{Kg} / \mathrm{m}^{2}$ & $26.22 \pm 5.42$ & & $26.32 \pm 4.46$ & & 0.916 \\
\hline Hypertension & Yes & 7 & 20 & 19 & 17.8 & 0.766 \\
\hline Diabetes mellitus & Yes & 4 & 11.4 & 8 & 7.5 & 0.49 \\
\hline Smoking status & Yes & 19 & 54.3 & 50 & 46.7 & 0.437 \\
\hline \multirow[t]{2}{*}{ Lauren classification } & Intestinal & 32 & 91.4 & 88 & 82.2 & 0.192 \\
\hline & Diffuse & 3 & 8.6 & 19 & 17.8 & \\
\hline \multirow[t]{5}{*}{ Location } & GEJ & 4 & 11.4 & 4 & 3.7 & 0.511 \\
\hline & Cardia & 14 & 40 & 47 & 43.9 & \\
\hline & Body & 8 & 22.9 & 24 & 22.4 & \\
\hline & Antrum & 9 & 25.7 & 28 & 26.2 & \\
\hline & Linitis plastica & 0 & 0 & 4 & 3.7 & \\
\hline \multirow[t]{3}{*}{ Histology } & SRCC & 7 & 20 & 17 & 15.9 & 0.151 \\
\hline & $\mathrm{AC}$ & 27 & 77.1 & 74 & 69.2 & \\
\hline & MAC & 1 & 2.9 & 16 & 15 & \\
\hline \multirow[t]{3}{*}{ Grade } & I & 4 & 11.4 & 8 & 7.6 & 0.336 \\
\hline & II & 24 & 68.6 & 63 & 60 & \\
\hline & III & 7 & 20 & 34 & 32.4 & \\
\hline \multirow[t]{3}{*}{ NACT Regimen } & EOF-EOX-ECF-ECX & 12 & 34.3 & 40 & 37.4 & 0.797 \\
\hline & DCX-DCF & 8 & 22.9 & 19 & 17.8 & \\
\hline & FLOT & 15 & 42.9 & 48 & 44.9 & \\
\hline NACT cycle no. & & $3.8 \pm 2.2$ & & $3.4 \pm 1.12$ & & 0.17 \\
\hline \multirow[t]{2}{*}{ Gastrectomy } & Subtotal & 7 & 20 & 16 & 15 & 0.482 \\
\hline & Total & 28 & 80 & 91 & 85 & \\
\hline \multirow[t]{2}{*}{ Lymphadenectomy } & D1 & 26 & 74.3 & 68 & 63.6 & 0.244 \\
\hline & D2 & 9 & 25.7 & 39 & 36.4 & \\
\hline Surgical margin & positive & 0 & 0 & 10 & 9.3 & 0.061 \\
\hline \multirow[t]{4}{*}{ урTNM } & 0 & 12 & 34.3 & 0 & 0 & $<0.001$ \\
\hline & 1 & 16 & 45.7 & 2 & 1.9 & \\
\hline & 2 & 6 & 17.1 & 30 & 28 & \\
\hline & 3 & 1 & 2.9 & 75 & 70.1 & \\
\hline \multirow[t]{5}{*}{ урт } & 0 & 12 & 34.3 & 0 & 0 & $<0.001$ \\
\hline & 1 & 11 & 31.4 & 6 & 5.6 & \\
\hline & 2 & 6 & 17.1 & 5 & 4.7 & \\
\hline & 3 & 6 & 17.1 & 64 & 59.8 & \\
\hline & 4 & 0 & 0 & 32 & 29.9 & \\
\hline \multirow[t]{4}{*}{ ypN } & 0 & 32 & 91.4 & 13 & 12.1 & $<0.001$ \\
\hline & 1 & 1 & 2.9 & 29 & 27.1 & \\
\hline & 2 & 2 & 5.7 & 20 & 18.7 & \\
\hline & 3 & 0 & 0 & 45 & 42.1 & \\
\hline No. of nodes removed & & $23.6 \pm 10.2(9-51)$ & & $28.6 \pm 13.8(10-78)$ & & 0.08 \\
\hline
\end{tabular}


Table 2. Continued

\begin{tabular}{|c|c|c|c|c|c|c|}
\hline \multirow[t]{2}{*}{ Variable } & & \multicolumn{2}{|c|}{ Group $1(\mathrm{n}=35)$} & \multicolumn{2}{|c|}{ Group $2(n=107)$} & \multirow[t]{2}{*}{$\mathrm{p}$} \\
\hline & & $\mathrm{n}$ & $\%$ & $\mathrm{n}$ & $\%$ & \\
\hline No. Of nodes positive & & $0.26 \pm 0.93(0-5)$ & & $6.66 \pm 7.28(0-35)$ & & $<0.001$ \\
\hline PNI & Presence & 5 & 14.3 & 72 & 67.3 & $<0.001$ \\
\hline LVI & Presence & 2 & 5.7 & 74 & 69.2 & $<0.001$ \\
\hline \multirow[t]{4}{*}{ HER-2 status } & 0 & 25 & 71.4 & 83 & 77.6 & 0.468 \\
\hline & 1 & 3 & 8.6 & 9 & 8.4 & \\
\hline & 2 & 5 & 14.3 & 7 & 6.5 & \\
\hline & 3 & 2 & 5.7 & 8 & 7.5 & \\
\hline \multirow[t]{5}{*}{ TRG } & I & 12 & 34.3 & 0 & 0 & $<0.001$ \\
\hline & II & 23 & 65.7 & 0 & 0 & \\
\hline & III & 0 & 0 & 44 & 41.1 & \\
\hline & IV & 0 & 0 & 58 & 54.2 & \\
\hline & $\mathrm{V}$ & 0 & 0 & 5 & 4.7 & \\
\hline \multirow[t]{4}{*}{ ACT regimen } & XELOX-FOLFOX-CF & 5 & 14.3 & 30 & 28 & 0.401 \\
\hline & EOX-EOF-ECX-ECF & 11 & 31.4 & 32 & 29.9 & \\
\hline & DCF-DCX & 6 & 17.1 & 15 & 14 & \\
\hline & FLOT & 13 & 37.1 & 30 & 28 & \\
\hline \multirow[t]{8}{*}{ Recurrence and localization } & yes & 4 & 11.4 & 48 & 44.9 & $<0.001$ \\
\hline & locoregional & 0 & 0 & 2 & 4.2 & \\
\hline & liver & 1 & 25 & 15 & 31.3 & \\
\hline & Peritoneum & 0 & 0 & 20 & 41.7 & \\
\hline & Distant LN & 2 & 50 & 4 & 8.3 & \\
\hline & Lung & 1 & 25 & 4 & 8.3 & \\
\hline & Brain & 0 & 0 & 1 & 2.1 & \\
\hline & bone & 0 & 0 & 2 & 4.2 & \\
\hline Last Status & Exitus & 0 & 0 & 23 & 21.5 & 0.003 \\
\hline
\end{tabular}

Abbreviations: See Table 1.

Table 3. Multivariate Analysis for TRG Groups (TRG I-II vs. III-IV-V).

\begin{tabular}{llccc}
\hline Variable & & OR & $95 \%$ CI for OR & $\mathrm{p}$ \\
\hline Age & year & 0.975 & $0.916-1038$ & 0.427 \\
Height & $\mathrm{cm}$ & 0.897 & $0.800-1004$ & 0.058 \\
Weight & $\mathrm{kg}$ & 1.023 & $0.922-1.134$ & 0.667 \\
BMI & $<25 \mathrm{~kg} / \mathrm{m}^{2}$ (ref.) & & 0.369 \\
& $25-30 \mathrm{~kg} / \mathrm{m}^{2}$ & 0.61 & $0.085-4.362$ & 0.623 \\
& $>25 \mathrm{~kg} / \mathrm{m}^{2}$ & 0.125 & $0.004-3.617$ & 0.226 \\
Lauren classification & Diffuse vs Intestinal & 1.423 & $0.210-9.600$ & 0.717 \\
Histology & SRCC(Ref.) & 1 & & 0.314 \\
& AC & 3.734 & $0.653-21.332$ & 0.138 \\
NACT cycle no. & MAC & 4.384 & $0.250-76.750$ & 0.312 \\
PNI & & 0.856 & $0.605-1.210$ & 0.379 \\
LVI & positive vs Negative & 5.318 & $1.194-23.681$ & 0.028 \\
\hline Abrit & positive vs Negative & 25.098 & $4.361-144.416$ & $<0.001$ \\
\hline
\end{tabular}

Abbreviations: See Table 1.

lymph nodes to the total number of lymph nodes removed was similar between the BMI groups. In our study, BMI did not affect TRG. Similar to previous studies, we observed that BMI did not affect DFS and OS.

In the study with $264 \mathrm{GC}$ patients by $\mathrm{Xu}$ et al., it was reported that TRG was correlated with both DFS and OS. Lauren classification and ypT were the independent factors for TRG (Xu et al., 2019). Zhu et al. reported that Mandard TRG system had correlation with survival in GC patients treated with NACT. In their study, TRG 
systems were significantly correlated with tumor grade, stage, LVI, PNI, and tumor size (Zhu et al., 2017). Smyth EC et al. conducted a randomized trial with GC patients and reported that TRG was in correlation with survival. In this study, mOS could not be reached in patients with TRG I-II, whereas mOS was 20 months in patients with TRG III-IV-V. Additionally, pathologic response to NACT was not correlated with any clinicopathological variable, including sex, age, tumor location, or histologic type (Smyth et al., 2016). Similar to these studies, in our study, TRG was correlated with survival. In our study, mDFS was 46 months in TRG I-II, while it was 18 months in TRG IV-V. Likewise, the TRG response was correlated with OS. Moreover, the presence of PNI and LVI were determined as the factors affecting TRG in our study.

In the literature, we could not find any study that examined the effect of BMI on TRG. Unlike the other studies, our study included a more heterogeneous patient group. We included only clinical stage III patients in our study. However, there were some limitations in our study. Our study was single-centered and had a retrospective nature. Since there were only 3 patients with BMI $<18$ in our study, they were not evaluated as a separate group.

In conclusion, we found that pre-treatment obesity status did not affect the response to NACT or long-term survival in clinical stage III GC patients. However, presence of PNI and LVI were determined as the factors negatively affecting response to treatment. In our study, DFS and OS were significantly greater as the response to NACT increased.

\section{Main Points}

- The relation of obesity with postoperative complications and survival in solid cancers were examined in many studies

- The effect of obesity on response to neoadjuvant chemotherapy(NACT) in Gastric cancer(GC) remains unknown.

- In the study, we found that pre-treatment obesity status did not affect the response to NACT or long-term survival in clinical stage III GC patients.

- Presence of perineural invasion and lymphovascular invasion affected the response to NACT.

- Survival was significantly greater as the response to NACT increased

\section{Acknowledgments}

Institutional review board statement: This study and all relevant procedures were performed in accordance with the Helsinki Declaration after obtaining the ethical board approval from the Van Yüzüncü Yıl University Ethics Committee (2020/03-52).

Informed consent statement: The patients were not required to give informed consent for this study because the study utilized the anonymous retrospective data obtained after each patient accepted the treatment by a written consent.

\section{Author Contributions}

Concept - AS, MNA, AbS; Design - AS, MNA, CK,
SS; Supervision - AS, MNA, SS, AbS; Resources -SS, AbS, IB, CK; Materials - AS, MNA, IB; Data Collection and/or Processing - SS, AbS, IB, CK; Analysis and/or Interpretation - AS, MNA, IB, CK; Literature Search - SS, AbS, IB; Writing Manuscript - AS, SS; Critical Review - SS, AbS, IB, CK; Other - AbS, IB, CK.

\section{Conflict-of-interest statement}

All authors declare no conflicts-of-interest related to this article.

\section{References}

Al-Batran SE, Hofheinz RD, Pauligk C, et al (2016). Histopathological regression after neoadjuvant docetaxel, oxaliplatin, fluorouracil, and leucovorine versus epirubicin, cisplatin, and fluorouracil or capecitabine in patients with resectable gastric or gastro-esophageal junction adenocarcinoma (FLOT4-AIO): results from the phase 2 part of a multicenter, open-label, randomized phase $2 / 3$ trial. Lancet Oncol, 17, 1697-708.

Al-Batran SE, Homann N, Pauligk C, et al (2019). Perioperative chemotherapy with fluorouracil plus leucovorine, oxaliplatin, and docetaxel versus fluorouracil or capecitabine plus cisplatin and epirubicin for locally advanced, resectable gastric or gastro-esophageal junction adenocarcinoma (FLOT4): a randomized, phase 2/3 trial. Lancet, 393, 1948-57.

Bege T, Lelong B, Francon D, et al (2009). Impact of obesity on short-term results of laparoscopic rectal cancer resection. Surg Endosc, 23, 1460-4.

Benns M, Woodall C, Scoggins C, et al (2009). The impact of obesity on outcomes following pancreatectomy for malignancy. Ann Surg Oncol, 16, 2565-9.

Bickenbach KA, Denton B, Gonen M, et al (2013). Impact of obesity on perioperative complications and long-term survival of patients with gastric cancer. Ann Surg Oncol, 20, 780-7.

Cunningham D, Allum WH, Stenning SP, et al (2006). Perioperative chemotherapy versus surgery alone for resectable gastroesophageal cancer. $N$ Engl J Med, 355, 11-20.

Dhar DK, Kubota H, Tachibana M, et al (2000). Body mass index determines the success of lymph node dissection and predicts the outcome of gastric carcinoma patients. Oncology, 59, 18-23.

Duconseil P, Garnier J, Weets V, et al (2019). Effect of clinical status on survival in patients with borderline or locally advanced pancreatic adenocarcinoma. World J Surg Oncol, 17, 95.

Farr A, Stolz M, Baumann L, et al (2017). The effect of obesity on pathological complete response and survival in breast cancer patients receiving uncapped doses of neoadjuvant anthracycline-taxane-based chemotherapy. Breast $J, 33$, 153-8.

Ferlay J, Soerjomataram I, Dikshit R, et al (2015). Cancer incidence and mortality worldwide: sources, methods and major patterns in GLOBOCAN 2012. Int $J$ Cancer, 136, E359-86.

Karatas F, Erdem GU, Sahin S, et al (2017). Obesity is an independent prognostic factor of decreased pathological complete response to neoadjuvant chemotherapy in breast cancer patients. Breast $J$, 32, 237-44.

Kobayashi T, Kimura T (2000). [Long-term outcome of preoperative chemotherapy with 5'-deoxy-5-fluorouridine (5'-DFUR) for gastric cancer]. Gan To Kagaku Ryoho, 27, 
1521-6.

Kunisaki C (2010). Reply to 090220: re: 'predictive factors for surgical complications of laparoscopy-assisted distal gastrectomy for gastric cancer' (Epub 31 Dec 08). Surg Endosc, 24, 247.

Kunisaki C, Makino H, Takagawa R, et al (2009). Predictive factors for surgical complications of laparoscopy-assisted distal gastrectomy for gastric cancer. Surg Endosc, 23, 2085-93.

Li Z, Shan F, Wang Y, et al (2018). Correlation of pathological complete response with survival after neoadjuvant chemotherapy in gastric or gastroesophageal junction cancer treated with radical surgery: A meta-analysis. PLoS One, 13, e0189294.

Li ZY, Koh CE, Bu ZD, et al (2012). Neoadjuvant chemotherapy with FOLFOX: improved outcomes in Chinese patients with locally advanced gastric cancer. J Surg Oncol, 105, 793-9.

Liedman B, Andersson H, Berglund B, et al (1996). Food intake after gastrectomy for gastric carcinoma: the role of a gastric reservoir. Br J Surg, 83, 1138-43.

Lorenzen S, Thuss-Patience P, Al-Batran SE, et al (2013). Impact of pathologic complete response on disease-free survival in patients with esophagogastric adenocarcinoma receiving preoperative docetaxel-based chemotherapy. Ann Oncol, 24, 2068-73.

Melcher AA, Mort D, Maughan TS (1996). Epirubicin, cisplatin and continuous infusion 5-fluorouracil (ECF) as neoadjuvant chemotherapy in gastro-oesophageal cancer. Br J Cancer, 74, 1651-4.

Mokdad AH, Ford ES, Bowman BA, et al (2003). Prevalence of obesity, diabetes, and obesity-related health risk factors, 2001. JAMA, 289, 76-9.

Palmela C, Velho S, Agostinho L, et al (2017). Body composition as a prognostic factor of neoadjuvant chemotherapy toxicity and outcome in patients with locally advanced gastric cancer. $J$ Gastric Cancer, 17, 74-87.

Park IJ, You YN, Skibber JM, et al (2017). Oncologic and functional hazards of obesity among patients with locally advanced rectal cancer following neoadjuvant chemoradiation therapy. Am J Clin Oncol, 40, 277-82.

Siegel RL, Miller KD, Jemal A (2019). Cancer statistics, 2019. CA Cancer J Clin, 69, 7-34.

Smyth EC, Fassan M, Cunningham D, et al (2016). Effect of pathologic tumor response and nodal status on survival in the medical research council adjuvant gastric infusional chemotherapy trial. J Clin Oncol, 34, 2721-7.

Sun L, Xu T, Yuan X, et al (2020). Obesity is a predictor in prostate cancer patients receiving prostatectomy after neoadjuvant hormonal therapy. Tumori, 106, 133-8.

Tsujinaka T, Sasako M, Yamamoto S, et al (2007). Influence of overweight on surgical complications for gastric cancer: results from a randomized control trial comparing D2 and extended para-aortic D3 lymphadenectomy (JCOG9501). Ann Surg Oncol, 14, 355-61.

Wong J, Rahman S, Saeed N, et al (2014). Effect of body mass index in patients undergoing resection for gastric cancer: a single center US experience. J Gastrointest Surg, 18, 505-11.

Xiong BH, Cheng Y, Ma L, et al (2014). An updated meta-analysis of randomized controlled trial assessing the effect of neoadjuvant chemotherapy in advanced gastric cancer. Cancer Invest, 32, 272-84.

Xu X, Zheng G, Zhang T, et al (2019). Is pathologic tumor regression grade after neo-adjuvant chemotherapy a promising prognostic indicator for patients with locally advanced gastric cancer? A cohort study evaluating tumor regression response. Cancer Chemother Pharmacol, 84, $635-46$.
Zhu Y, Sun Y, Hu S, et al (2017). Comparison of five tumor regression grading systems for gastric adenocarcinoma after neoadjuvant chemotherapy: a retrospective study of 192 cases from National Cancer Center in China. BMC Gastroenterol, 17, 41.

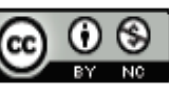

This work is licensed under a Creative Commons AttributionNon Commercial 4.0 International License. 